\title{
Practical approximations and heuristic approaches for managing shiftable loads in the multi-period optimal power flow framework
}

\author{
Iason-Iraklis Avramidis ${ }^{\mathrm{a}, \mathrm{b}}$, Florin Capitanescu ${ }^{\mathrm{a}, *}$, Geert Deconinck ${ }^{\mathrm{b}}$ \\ ${ }^{a}$ Luxembourg Institute of Science and Technology (LIST), Environmental Research and \\ Innovation (ERIN) Department, 41-rue du Brill, L-4422 Belvalux, Luxembourg \\ ${ }^{b}$ ESAT-Electa, KU Leuven, Kasteelpark Arenberg 10, Leuven 3001, Belgium
}

\begin{abstract}
The presence of smart buildings (SBs) is expected to grow manifold in the next decade, leading to both challenges and opportunities in managing increasingly "active" distribution systems. SBs are host to a number of devices and technologies capable of providing residential flexibility in order to maintain acceptable operating conditions within the distribution system. A prevalent class of devices with significant potential for flexibility provision are shiftable loads (SLs), which represent a large amount of potential SB-provided flexibility. However, the accurate modelling of SLs within the multi-period optimal power flow (MP-OPF) framework results in complex non-convex mixed-integer nonlinear programming (MINLP) problems. For realistic feeders, MINLP problems are intractable and hence require some heuristic form of circumvention. This work evaluates the limitations of the MINLP formulation and proposes new practical alternatives, namely a flexible NLP approximation and a multi-faceted heuristic algorithm. The proposed approaches are generic and applicable for any problem size or type of SL, while they are shown to work efficiently, both speed-wise and solutionquality-wise, outperforming state-of-the-art MINLP solvers. A simple scoring and ranking scheme is also proposed in order to make a direct comparison between the different approaches.
\end{abstract}

Keywords: mixed-integer nonlinear programming, multi-period optimal

\footnotetext{
${ }^{*}$ Corresponding author

Email address: fcapitanescu@yahoo.com (Florin Capitanescu)
} 
power flow, residential flexibility, shiftable loads, smart buildings

\section{Nomenclature}

\section{Sets}

$\mathcal{I} \quad$ Set of nodes

$\mathcal{P} \quad$ Set of photovoltaic (PV) panels

$\mathcal{S} \quad$ Set of shiftable load (SL) groups

$\mathcal{T} \quad$ Set of time periods

Parameters: Power system quantities

$b / g_{i j} \quad$ Susceptance/conductance of branch $i-j, \mathrm{pu}$

$C T_{s} \quad$ Cycle time of SL $s$, time periods

$I_{i j}^{\max } \quad$ Line $i-j$ current limit, $\mathrm{pu}$

$D_{i, t}^{P, Q} \quad$ Active/reactive load demand at bus $i$, period $t, \mathrm{pu}$

$P_{s}^{\text {rate }}$ Average rated power of SL group $s, \mathrm{pu}$

$P_{p, t}^{g e n} \quad \mathrm{PV} p$ generated active power, period $t, \mathrm{pu}$

$S_{i j}^{m a x} \quad$ Transformer $i-j$ apparent power limit, pu

$t_{s}^{0} \quad$ Time period $t$ when SL $s$ would normally turn "ON"

\section{Parameters: Monetary values}

$c^{I E} \quad$ Price of import/export from/to the MV level, $€ / \mathrm{kWh}$

$c^{P V} \quad$ Price of PV production curtailment, $€ / \mathrm{kWh}$

$c^{S L} \quad$ Price of SL commitment and shifting, $€ / \mathrm{kW}$

$c^{\text {viol }}$ Penalty for technical limits violation, $€ / \mathrm{pu}$

\section{Variables}


$E C_{t}^{I E}$ Cost of energy import/export from/to the MV level, period $t, €$

$E C_{t}^{P V}$ Cost of PV production curtailment, period $t, €$

$C C^{S L}$ Cost of SL commitment and shifting, $€$

$V C_{t} \quad$ Cost for limits violation, period $t, €$

$P_{i j, t} \quad$ Branch $i-j$ active power flow, period $t, \mathrm{pu}$

$I E_{t}^{P, Q}$ Active/reactive imports/exports, period $t, \mathrm{pu}$

$P_{p, t}^{P V} \quad \mathrm{PV} p$ injected active power, period $t, \mathrm{pu}$

$Q_{i j, t} \quad$ Branch $i-j$ reactive power flow, period $t, \mathrm{pu}$

$V_{i, t} \quad$ Voltage magnitude at bus $i$, period $t, \mathrm{pu}$

$\delta_{s, t} \quad$ Binary variable for SL $s$, period $t$. It is equal to 1 if the SL is "ON"; otherwise it is equal to 0 .

$\theta_{i j, t} \quad$ Voltage angle difference of buses $i, j$, period $t, \mathrm{pu}$

$\sigma_{i, t}^{u / d} \quad \mathrm{Up} /$ down voltage violation at bus $i$, period $t, \mathrm{pu}$

$\sigma_{i j, t} \quad$ Branch $i-j$ thermal limit violation, period $t, \mathrm{pu}$

\section{Introduction}

\subsection{Motivation and literature review}

Distribution systems have begun hosting an increasing number of renewable energy technologies and "smart" devices [1]. However, the uncoordinated operation of customer-owned distributed energy resources (DER) can lead to operational issues, i.e., voltage/thermal limits violations, which seriously jeopardize reliable customer service [2]. The need to identify devices with high constraint management potential is crucial.

Residential smart buildings (SBs) have been identified as potential providers of flexibility for combating operational issues in distribution grids [3]. Besides causing (on average) less local stress to the system, SBs are excellent sources of concentrated flexibility, being able to provide a variety of services for combating operational issues. In that light, they are rendered more effective than individual DER scattered throughout the system. 
The envisioned SBs contain a common class of devices capable of providing significant residential flexibility: shiftable (or deferable) loads (SLs). The term describes any device whose operation can be shifted to different time periods (in service of some objective). Said category includes (among others) washing machines, dryers, freezers or boilers. Depending on their nature, SLs can be categorized as interruptible or uninterruptible [4].

The optimal management of SLs has been tackled by recent research works. The approach almost exclusively employed in MP-OPF formulations is constructing simplified versions of SLs, usually assuming a single consumption level for the optimization horizon and ignoring the modelling of transitional periods [5], [6], [7]. These approaches often disregard the costs associated with the provision of flexibility. A common alternative is clustering multiple SL owners before proceeding to any optimization. Heuristic algorithms are usually employed for estimating individual SL profiles, see [8].

In MP-OPF settings, SLs often correspond to the aggregate behavior of all shiftable devices within a household. The authors of [5] employ auxiliary variables to model the switching behavior of SLs in a MILP setting. This is extended in [6], where an incentive-based scheme is developed, that also includes constraints on acceptable operation time-frames. In [7], the problem is extended to the MINLP setting, where several building management systems and electric vehicles charging stations are optimally coordinated by an aggregator. The authors of [9] use dynamic pricing to combine SL scheduling with PV production uncertainty, allowing for almost unrestricted profile alteration. The concept of in-building load shifting is explored in [10], where device-specific constraints are included.

When pure mathematical modelling is employed, large problems can easily grow intractable; several approaches have been proposed to address this. Assuming substantial communication infrastructure between energy suppliers, load aggregators and individual households, machine learning has been proposed for aggregating and managing SLs under various strategies [11][12]. The paper [13] describes a local control algorithm in a dynamic pricing environment, where demand response-motivated offers are made on flexible amounts of shiftable demand. Ref. [4] decides on the scheduling of SLs by mapping objective values to different scheduling scenarios. In [14], an aggregator employs a closed-loop control scheme, where a reference signal is used for controlling an SL group.

Meta-heuristics and probabilistic approaches have also received some attention in the past. Examples include [15], where the cuckoo search algorithm 
is employed for optimal SL management without resorting to SL modelling, and [16], where SL modelling is avoided by employing a probabilistic scheduling algorithm based on sophisticated smart meters.

\subsection{Contributions and paper organization}

The optimal coordination of several SLs to manage power system operational issues on larger scales is an underaddressed issue, especially when each individual customer impact is considered. Very few scalable and reliable approaches have been proposed. This is the research gap that this work addresses, within the general MP-OPF framework (based on the original work of [17]). The authors have already performed some preliminary research on the topic [18], recording the behavior of smaller-scale systems hosting SLs. This work significantly expands upon said previous work.

This paper's main contributions are the following:

- A flexible NLP approximation for SL modelling, validated on realisticsize distribution feeders. The SL management problem is additionally complemented by a novel commitment cost, associated with SL rescheduling.

- A heuristic algorithm to estimate the positioning of multiple SLs and subsequently solve MINLP distribution system management problems. Furthermore, the technique on which the heuristic algorithm is based provides valuable information regarding the impact of each SL behavioral aspect in determining its operation.

The remainder of this paper is structured as follows. Section 2 describes the MP-OPF problem formulation. Section 3 analyzes the proposed heuristic

algorithm. Section 4 presents the case studies. Conclusions are laid out in Section 5 .

\section{Day-ahead planning problem: MP-OPF formulation}

\subsection{Day-ahead vs real-time management}

This paper deals with the day-ahead planning of power system problem. In general, power system operation planning is performed in two main steps: day-ahead planning and real-time control (mostly automatic, through local/global control loops, and seldom operator-initiated). However, due to computation bottlenecks, risk of failure, physical/financial constraints on 
flexibility providers, and a prerequisite for processing massive measurements, the real time control (other than by existing local-rule-based control) is challenging and the large experience in power system operation indicates that operators (prefer to) rely mostly on day-ahead planning actions. This almost exclusive reliance on day-ahead decisions/actions is even stronger in distribution systems, which today have little room for operator-initiated real-time control but would need to improve this aspect during the transition toward smart grids. While the ideal approach is the consideration of both aspects (which we plan to address thoroughly in future work), we neglect for now realtime (operator-initiated) control and focus on a cautious day-ahead planning, approach which is aligned with many research papers (see e.g., [19, 20, 21, 22] for further information).

In addition, the focus on the planning process also stems from technical factors. With the development of more sophisticated forecasting tools, the forecasting errors are significantly alleviated, especially on distribution systems thanks to the limited geographical span. The distribution system operator (DSO) would have reasonably good knowledge of future developments (at least for shorter-term decisions), placing much heavier emphasis on the planning process.

In terms of usability of the strategy proposed, our MP-OPF computes optimal set-points for DERs and particularly time shifts for shiftable loads that are to be procured and financially agreed the day-ahead. The shiftable loads have then to follow during the operation of the next day the agreed schedules with the DSO. This is most commonly performed by a home energy management system.

\subsection{Assumptions and limitations}

\subsubsection{Power system level}

The following apply regarding all examined power system models:

- The feeder is assumed to be managed by some entity, e.g., an aggregator, that explicitly represents the DSO's primary interest, i.e., maintaining acceptable operating conditions across the optimization horizon. To achieve this, the aggregator may send curtailment signals to PVs or re-positioning signals to SLs, subject to customer remuneration. Note that this setup concerns future distribution feeders, burdened by operational issues and requiring the establishment of a framework aimed explicitly at alleviating said issues. 
- Measurement units and appropriate communication links are assumed to be pre-installed in the feeder. As such, the operational state of the feeder is well observable, and a centralized day-ahead optimization is possible by transmitting new operational set-points.

- For simplicity, the presented work focuses on balanced distribution systems in a deterministic setting. It must be mentioned that a) the approach is generic and readily extendable to unbalanced settings and b) stochasticity is not the current focus, but is a future research avenue.

\subsubsection{Shiftable load level}

The following apply regarding all examined shiftable loads:

- Modelling all household SLs (using multiple MI formulations) is extremely inefficient. The computational burden would drastically increase, with little to no avail (a single SL has negligible impact, even locally). In this paper, all SLs are grouped into a single entity with a specific rated power, cycle time and standard operation, this being the most common approach employed when examining problems at the system level. The differences between the simplified approach and a more detailed one are not meaningful enough. As such, detailed modelling of all SLs is not practically warranted.

- When SLs share some similarities (usually geographical location), clustering is commonly employed. This grouping could be a local energy community, an aggregator-managed area, or an area of interest for the DSO. While clustering is a good technique for employing residential flexibility on larger scales, it is impractical within the paper's framework. Within the paper's scope, SLs create their own local issues; it is much more efficient to target specific controllable resources rather than resorting to system-wide measures. In case a common profile was employed for multiple SLs, said profile would subsequently have to be "split" between the grouped SLs, adding further complexity.

\subsection{Mathematical modelling}

\subsubsection{Objective function}

A day-ahead centralized control scheme is considered for scheduling controllable devices. The main objective, across the optimization horizon, is 
achieving cost-optimal and issue-free operation for the feeder. Without loss of generality, we consider three hourly optimization horizons (6, 12, 24 hours).

The objective function (1) represents the total management cost, including the import/export (from the MV level) cost $E C_{t}^{I E}(2)$, the PV curtailment cost $E C_{t}^{P V}(3)$, the SL commitment and shifting cost $C C^{S L}$ (4) and the slack violations cost $V C_{t}(5)$ :

$$
\begin{array}{r}
\min \left\{\sum_{t=1}^{T}\left(E C_{t}^{I E}+E C_{t}^{P V}+V C_{t}\right)+C C^{S L}\right\} \\
E C_{t}^{I E}=I E_{t}^{P} \cdot c^{I E} \quad \forall t \in \mathcal{T} \\
E C_{t}^{P V}=\sum_{p \in \mathcal{P}}\left(P_{p, t}^{g e n}-P_{p, t}^{P V}\right) \cdot c^{P V} \quad \forall t \in \mathcal{T} \\
C C^{S L}=\sum_{s \in \mathcal{S}} P_{s}^{r a t e} \cdot\left(1-\delta_{s, t_{s}^{0}} \cdot \delta_{s,\left(t_{s}^{0}+C T_{s}\right)}\right) \cdot c^{S L} \\
V C_{t}=\sum_{i, j \in \mathcal{I}: i \neq j}\left(\sigma_{i, t}^{u}+\sigma_{i, t}^{d}+\sigma_{i j, t}\right) \cdot c^{v i o l} \quad \forall t \in \mathcal{T}
\end{array}
$$

Energy imports/exports from/to the MV level $I E_{t}^{I E}$ are proportionally priced (cost of $c^{I E}=10$ ). The power generation of PVs can be curtailed (at inverter level), in which case their owners are reimbursed (cost of $c^{P V}=$ 25). Voltage and thermal technical limits violations $\sigma_{i, t}^{u}, \sigma_{i, t}^{d}, \sigma_{i j, t}$ are heavily penalized in (cost of $c^{\text {viol }}=100$ ), though they can occur; this guarantees that a feasible solution can be obtained for comparison purposes. $c^{I E}, c^{P V}$ are originally expressed in terms of $€ / \mathrm{kWh}$, though they are converted to $€ / \mathrm{pu}$ (MP-OPF based on pu elements). $c^{\text {viol }}$ is originally expressed in $€ / \mathrm{pu}$.

As was mentioned, a novelty over previous works is the formulation of the SL rescheduling cost. If an SL starts/ends its operation earlier/later than the customer-defined directive, i.e., $\delta_{s,\left(t_{s}^{0}\right)}=0$ or $\delta_{s,\left(t_{s}^{0}+C T_{s}\right)}=0$, the customer is remunerated at a fixed commitment cost, proportional to the SL's rate and independent of how much the SL is shifted (4). This cost $\left(c^{S L}=30\right)$ is originally expressed in terms of $€ / \mathrm{kW}$, also converted to $€ / \mathrm{pu}$.

It should be mentioned that the chosen cost values do not reflect any real-life costs. They are chosen as such simply to reflect a desired activation priority order (PO) within the mathematical optimization framework, as is the standard approach in such cases. As stated already, the paper deals with "practical approximation and heuristic approaches for managing 
shiftable loads in the multi-period optimal power flow framework". Plainly put, the focus is on deciding the optimal planning of shiftable loads by devising clever alternatives to solving the computationally unmanageable MINLP problem. While not true for any application, as long as the PO is realistically represented, the impact on the current research issue is minimal, and the employed costs costs can be chosen (up to a degree) arbitrarily. Do note however that some degree of realism must still be maintained, so that the preliminary results present an outcome that can be practically interpreted.

It is the authors' intent to elaborate (in their future work) on how such costs would be practically determined, though only after validating their proposed approach. Even though the proposed formulation can then incorporate the more realistic costs, no direct links to real-life market values should be made at this stage.

\subsection{Technical constraints}

The generation of PVs $P_{p, t}^{g e n}$ can be curtailed up to a certain percentage, expressed by the parameter $M^{P V},(6)$, should it be deemed necessary to avoid overvoltages or line stressing. While the formulation can easily accommodate a modifiable power factor (pf) for PVs (reactive power suport), the pf is assumed to be 1, solely for simplicity:

$$
\left(1-M^{P V}\right) P_{p, t}^{g e n} \leq P_{p, t}^{P V} \leq P_{p, t}^{g e n} \quad \forall p \in \mathcal{P}, \forall t \in \mathcal{T}
$$

For the feeder, the classical power system constraints must also hold (7)(13), and do so $\forall t \in \mathcal{T}, \forall i, j \in \mathcal{I}: i \neq j, \forall p \in \mathcal{P}, \forall s \in \mathcal{S}$ :

$$
\begin{array}{r}
P_{i j, t}=-V_{i, t}^{2} g_{i j}+V_{i, t} V_{j, t}\left[g_{i j} \cos \theta_{i j, t}+b_{i j} \sin \theta_{i j, t}\right] \\
Q_{i j, t}=V_{i, t}^{2} b_{i j}+V_{i, t} V_{j, t}\left[g_{i j} \sin \theta_{i j, t}-b_{i j} \cos \theta_{i j, t}\right] \\
P_{i, p, t}^{P V}+I E_{t}^{P}-D_{i, t}^{P}-P_{i, s}^{r a t e} \cdot \delta_{s, t}=\sum P_{i j, t} \\
I E_{t}^{Q}-D_{i, t}^{Q}-Q_{i, s} \cdot \delta_{s, t}=\sum_{i \neq j, t} Q_{i j, t} \\
V_{i, t}^{\min }-\sigma_{i, t}^{d} \leq V_{i, t} \leq V_{i, t}^{\max }+\sigma_{i, t}^{u} \\
P_{i j, t}^{2}+Q_{i j, t}^{2} \leq\left(S_{i j, t}^{\max }\right)^{2}+\sigma_{i j, t} \\
P_{i j, t}^{2}+Q_{i j, t}^{2} \leq V_{i, t}^{2}\left(I_{i j, t}^{\max }\right)^{2}+\sigma_{i j, t}
\end{array}
$$


Constraints (7)-(8) are, respectively, the equations for the active and reactive branch power flows $P_{i j, t}, Q_{i j, t}$. For the nodal power balance constraints (9)-(10), $Q_{s}$ is the reactive power of SL group $s$. All fixed loads and SLs operate with a pf of 0.95. Constraints (11)-(13) describe the technical limitations on voltage magnitudes $V_{i, t}^{\min }, V_{i, t}^{\max }$, transformer power $S_{i j, t}^{\max }$ and line current $I_{i j, t}^{\max }$ limits respectively (relaxed by the inclusion of slack variables). All slack variables are positive.

\subsection{Modelling of Shiftable Loads}

\subsubsection{Discrete formulation}

In MP studies, SLs are modelled using discrete variables (here $\delta_{s, t}$ ) for representing their binary status ("ON" or "OFF") at each time interval. An SL's main requirement is fulfilling its energy mandate, i.e., the sum of all binary variables is equal to the SL's cycle time (CT) (14). In the case of uninterruptible SLs (whose operation may not halt before completing their $\mathrm{CT}$ ), an additional constraint must be placed to ensure that the first nonzero binary variable is followed by $C T_{s}-1$ non-zero binary variables. The SL thus fulfils the uninterrupted operation requirement or continuity mandate (15). Do note that (15) is in fact a set of constraints:

$$
\begin{array}{r}
\sum_{t=1}^{T} \delta_{s, t}=C T_{s} \quad \forall s \in \mathcal{S} \\
\sum_{k=t+1}^{t+C T_{s}} \delta_{s, t} \geq C T_{s} \cdot\left(\delta_{s, t+1}-\delta_{s, t}\right) \quad \forall t \in \mathcal{T}, \forall s \in \mathcal{S}
\end{array}
$$

\subsubsection{Continuous approximation}

Discrete SL modelling leads to intractability especially when considering large optimization horizons or multiple SLs. As such, a novel approximation approach is proposed as an alternative.

Constraint (14), i.e., the energy mandate, is kept in its original form (mandatory for all SLs). All binary variables, $\delta_{s, t}$ are relaxed to continuous variables within the range $[0,1]$. Ideally, all positive variables $\delta_{s, t}$ should be equal to one. Regardless of the SL's type, the number of positive $\delta_{s, t}$ is strictly limited by the SL's CT (16). This is hereby referred to as the limited activation mandate. This constraint limits the number of positive, formerly binary, variables by employing a barrier function. $\epsilon$ is the SL sensitivity factor 
and can be fine-tuned with respect to how accurate SLs modelling should be. It must be carefully selected to achieve adequate SL representation and to avoid potential numerical pitfalls, as was observed in [18].

$$
\sum_{t=1}^{T} \frac{\delta_{s, t}}{\delta_{s, t}+\epsilon} \leq C T_{s} \quad \forall s \in \mathcal{S}
$$

Again, an additional constraint is needed to model uninterruptible SLs. As such, the set of constraints (15) is now replaced by a single constraint (17); the pseudo-continuity mandate. Limits are placed on the (theoretical) cumulative ramp rate that the SL is allowed to have. This constraint limits the total change in value that the variables $\delta_{s, t}$ can have.

$$
\sum_{t=1}^{T-1} \delta_{s, t+1}\left(\delta_{s, t+1}-\delta_{s, t}\right) \leq 1 \quad \forall s \in \mathcal{S}
$$

Do note that, for the NLP approximation of uninterruptible SLs, no subset of the constraints (14), (16)-(17) can achieve the desired behavior. The three constraints work in a complementary manner, and the removal of any one would result in insufficient uninterruptible SL modelling.

\subsubsection{Remarks}

The MINLP MP-OPF (discrete SL) is described by (1)-(15). The NLP MP-OPF (continuous SL) is described by (1)-(14), (16)-(17).

It is worth pointing out that, for the original binary variables, the discrete and continuous formulations are equivalent, i.e., a MINLP solver produces the same results with both.

\section{Proposed heuristic approaches and scoring}

While mathematical SL models are desirable for MP-OPF studies, tractability is quickly lost for large systems/optimization horizons or when managing several SLs. Furthermore, NLP approximations of MINLP problems, although sophisticated, may present performance issues. Numerical stability and proper SL representation are major issues, and even if the convergence is fast, the solution quality may be worse than the MINLP one. 
These issues motivate us to propose an alternative to solving MINLP MP-OPF problems with a significant penetration of SLs. The most common approaches for dealing with SLs are heuristics. As such, we propose a flexible, multifaceted heuristic approach for managing SL-based intractability.

\subsection{Heuristic Approach: "fix-and-run"}

This work proposes a heuristic approach that builds upon the proposed NLP approximation. The main idea is to estimate (partially or fully) the behavior of each SL, force the estimate as a constraint ("fix") and simulate a more constrained version of the NLP approximation ("run"). The heuristic approach is thus named "heuristic fix-and-run".

\subsubsection{The rationale}

The mixed-integer formulation for SLs is made up of $T+1$ constraints (1 constraint stemming from Eq. (14) and $T$ constraints stemming from Eq. (15)). In transitioning to the nonlinear model for SLs, i.e., Eqs. (14), (16)(17), 3 constraints are instead employed. In each of the three constraints, even though the SL operation variables are continuous, their behaviour at the final solution very closely (almost perfectly) resembles the behaviour of binary variables.

Even though the nonlinear model is computationally lighter, it could still be subject to numerical issues, mainly due to Eq. (16). As such, some complementary heuristic is necessary. The main rationale behind the heuristic is the following.

The nonlinear SL model is composed of three aspects, all of which must be satisfied simultaneously in order to accurately reflect the desired behavior:

1. A requirement for all active operating periods of the SL to exactly match its cycle time, see (14).

2. A requirement for the (theoretical) cumulative ramp rate of the SL to be limited, in order to model only two operating modes (ON or OFF), see (17).

3. A requirement for the total number of all active operating periods of the SL to not be larger than its cycle time, see (16).

While all three aspects must hold in order for the nonlinear model to serve its purpose, an aspect could be more or less dominant than another, e.g., its removal could lead to small or large distortions of the final SL behavior produced by the nonlinear model. In attempting to study the characteristics 
of each aspect and essentially quantify its importance in the formulation, multiple different cases were examined, were different weights were given to each aspect. As such, we could explore (in a sense) the degree to which each of the three aforementioned constraints contribute to the final value of each originally binary variable. In other words, we proposed splitting the binary variable into three continuous forms and build its actual value (either 0 or 1) based on the composition of the three forms.

\subsubsection{The application}

The proposed approach consists of the following steps (per SL). First, one, two or three of the following versions (version denoted by $u$ ) of the NLP approximation are solved, where one of the three SL mandates is removed for each:

1. Version C: Ignore the pseudo-Continuity mandate (17).

2. Version A: Ignore the limited Activation mandate (16).

3. Version D: Ignore the energy Demand mandate (14).

Having obtained result from up to three versions, i.e., the values of the continuous variables $\delta_{s, t}^{v}$, we combine them into $\delta_{s, t}^{C M B}$, where CMB refers to "combination", using (18):

$$
\delta_{s, t}^{C M B}=\frac{\sum_{v \in\{C, A, D\}} \delta_{s, t}^{v} w^{v}}{\sum_{v \in\{C, A, D\}} w^{v}}
$$

The variables $w^{v}$ correspond to the desired weights of each NLP approximation version. Because it is unknown which of the three mandates is the most impactful from a modelling perspective, we must explore different combinations, from which we will draw conclusions regarding the constraints that can be reliably relaxed with minimal impact on the SLs' optimal profile. For a balanced perspective, the explored combinations cover a vast array of weighting choices. Examples of different combinations are presented in Table 1. Due to the methodology being generic, several different combinations (based on different values of $w^{v}$ ) can be developed.

Having obtained the values $\delta_{s, t}^{C M B}$ for any or all of the combinations, the optimal SL pattern can now be estimated. After the estimation, a complete NLP MP-OPF is run to determine the solution for the whole system. The estimation is based on the proposed "spot filling algorithm" (SFA). 


\subsection{Estimating the SL pattern}

This section presents the "spot filling algorithm" (SFA) for estimating the optimal pattern of SLs. The analysis that follows concerns a single combination and a single SL. The methodology is identical for all SLs and constructed combinations.

After solving the NLP approximation for a combinations, different consumption levels are calculated for the SL. For an optimization horizon of $T$ intervals and an SL with a CT of $X$ periods, one must determine an $X$ number of spots to take up the rated consumption value $P_{s}^{\text {rate }}$, while the remaining $T-X$ periods will represent the "OFF status".

This is performed in an iterative manner. At each iteration $i$, the time period with the highest consumption value $t_{i}^{C}$ is considered as the primary candidate for filling a spot in the SL profile. An evaluation is then performed with respect to the saved values of all previous iterations. If the minimum "time distance" $\triangle T D$ between the positions of the current value $t_{i}^{C}$ and the closest previously selected value $t_{i, a d j}^{C}$, is smaller than the number of remaining spots to be filled, $R S$, then the selected period $t_{i}$ as well as all periods between $t_{i}$ and $t_{i, a d j}^{C}$ are saved and an equal number of spots is filled up. Otherwise, the candidate value is discarded and we proceed to the next iteration. $\triangle T D$ and $t_{i, a d j}^{C}$ are calculated based on (19)-(20), where $\Omega$ is the dynamic set of all preceding iterations:

$$
\Delta T D= \begin{cases}t_{i}^{C}-\min \left(t_{k \in \Omega}^{C}\right) & \text {, if } t_{i}^{C}<\min \left(t_{k \in \Omega}^{C}\right) \\ t_{i}^{C}-\max \left(t_{k \in \Omega}^{C}\right) & , \text { if } t_{i}^{C}>\max \left(t_{k \in \Omega}^{C}\right)\end{cases}
$$

Table 1: Examples of versions of the proposed approach

\begin{tabular}{|c|c|c|c|c|}
\hline & \multicolumn{3}{|c|}{ Mandate ignored \& Assigned weights $w^{v}(\%)$} & \multirow[b]{2}{*}{$\begin{array}{l}\text { NLP versions } \\
\text { solved }\end{array}$} \\
\hline $\begin{array}{l}\text { Approach } \\
\text { code }\end{array}$ & $\begin{array}{c}\text { Continuity } \\
w^{C}\end{array}$ & $\begin{array}{c}\text { Limited activation } \\
w^{A}\end{array}$ & $\begin{array}{c}\text { Energy } \\
w^{D}\end{array}$ & \\
\hline $\mathrm{ES}$ & 33.3 & 33.3 & 33.3 & 3 \\
\hline C & 100 & 0 & 0 & 1 \\
\hline A & 0 & 100 & 0 & 1 \\
\hline D & 0 & 0 & 100 & 1 \\
\hline CAD & 60 & 30 & 10 & 3 \\
\hline $\mathrm{ACD}$ & 30 & 60 & 10 & 3 \\
\hline DCA & 30 & 10 & 60 & 3 \\
\hline
\end{tabular}




$$
t_{i, a d j}^{C}=t_{i}^{C}-\Delta T D
$$

Once all spots are filled, the corresponding time periods are set to a consumption level of $P_{s}^{\text {rate }}$, while the remaining are set to 0 . The complete NLP MP-OPF is then run to determine the final system solution. The SFA is presented as Algorithm 1.

For clarification, it should be pointed out that the solution of the NLP MP-OPF and the determination of the SL patterns (through Algorithm 1) occurs for all SLs simultaneously. Given that operational issues are best tackled by considering the synergy of the various controllable options (here SLs), determining the pattern of SLs with respect to how they interact with each

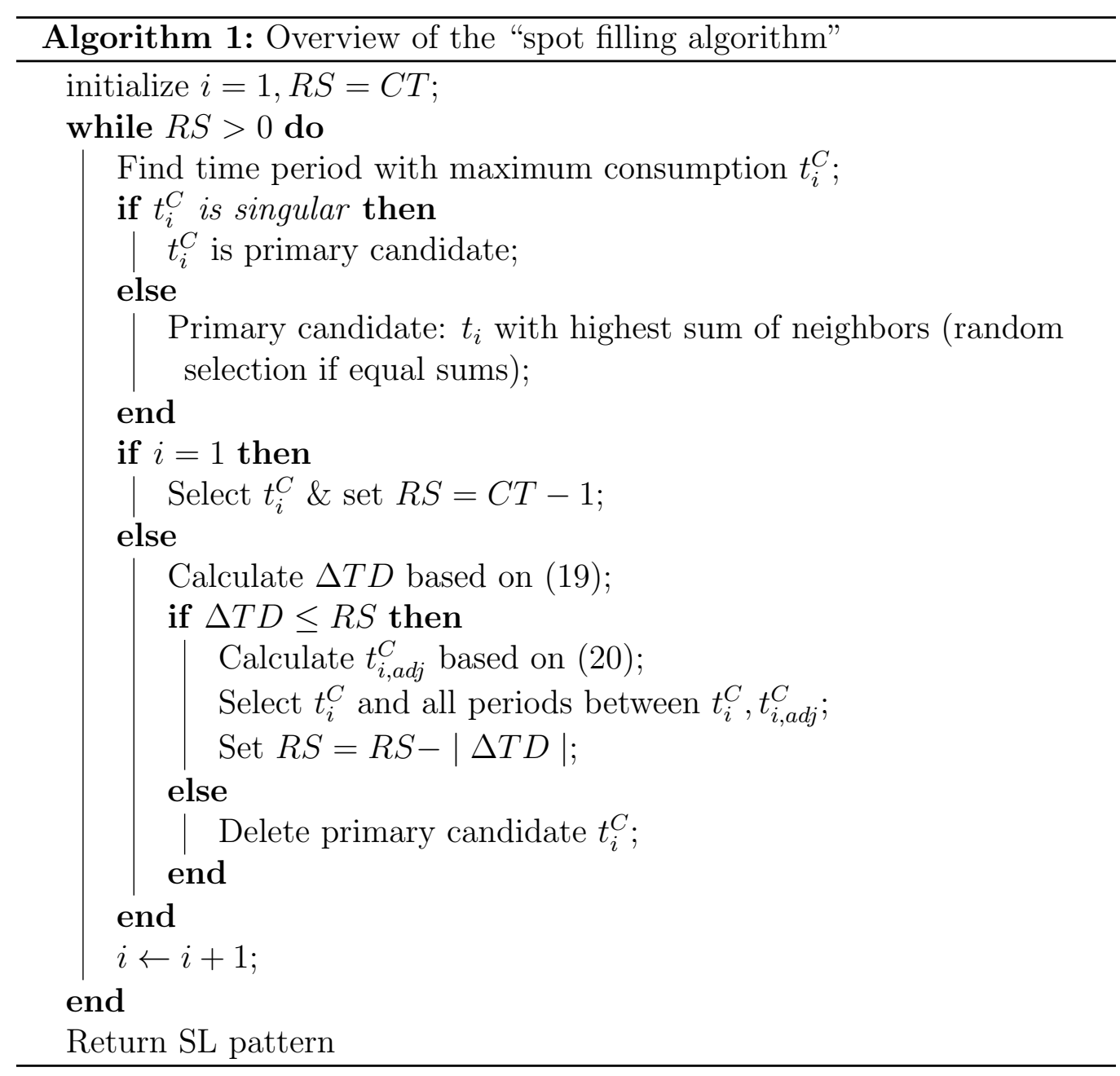


other is a preferable strategy. Besides, solving multiple NLPs (one per SL) and determining the pattern of each SL individually would be computationally inefficient and perhaps erroneous; attempting to combat system-wide violations by individual SLs could lead to significantly sub-optimal results. The process was described for a single SL on the grounds of simplicity and comprehension.

\subsection{Illustrative example}

For the sake of clarity, the authors present a simple illustrative example of the SFA. For this example, versions $A$ and $C$ of the heuristic approach are selected, each with an assigned weight of 0.5 . The pattern of a single SL must be determined, with that SL having a CT of 4 periods. An illustration of SFA's application for the described example is presented in Fig. 1.

After solving two different versions of the NLP approximation, the values of the relaxed variables $\delta_{s, t}^{u}$ are determined (upper left, blue color for approach $C$ and bottom left, brown color for approach $A$, where the red line represents the upper limit of one for the relaxed binary variables). Each set of values is multiplied by the assigned weight (0.5) and the resulting values summed up (summation in upper right, green color). We still have to estimate the SL pattern; four of the variables $\delta_{s, t}^{u}$ converted $1\left(C T_{s}=4\right)$, and the rest to 0 .

The first largest consumption value $C_{1}$ corresponds to period 6 . This fills the first spot by default, and reduces the remaining spots to 3 . In the second iteration, we identify the second largest consumption value $C_{2}$ as corresponding to period 5 . This is adjacent to the one selected in the previous iteration and is thus also acceptable; 2 empty spots remain.

In the third iteration, we identify the third highest value $C_{3}$ as corresponding to period 2. However, the distance between period 2 and its most adjacent period (5) is equal to 3 , which is larger than the number of remain-
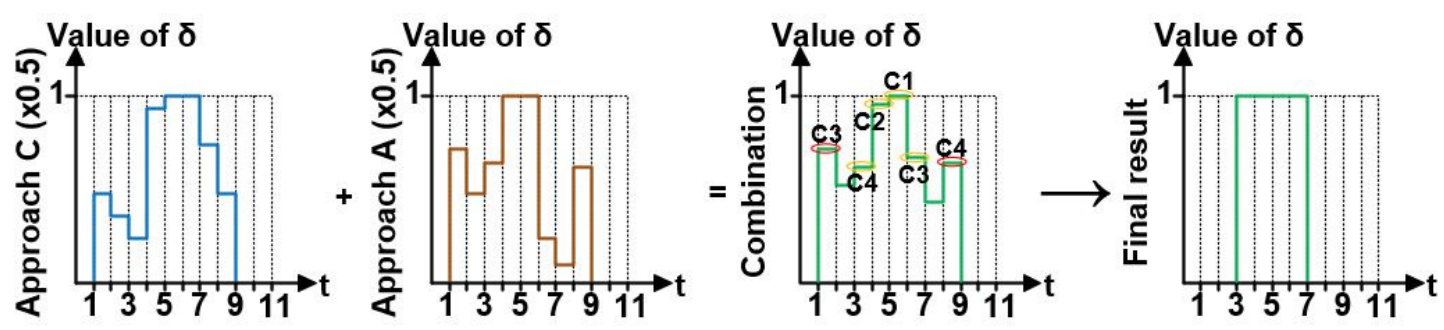

Figure 1: Heuristic algorithm visualization for the described example 
ing spots (2), and thus period 2 is unacceptable and discarded as a candidate. The next alternative for $C_{3}$ corresponds to period 7 , which is an acceptable option. In the next iteration, period 9 is similarly considered unacceptable to "host" the next highest consumption level $C_{4}$ and thus period 4 is chosen as the next best candidate. Having filled all the desired spots (4), the selected $\delta_{s, t}$ are set equal to one and the remaining are set equal to zero, with the desired SL profile having been estimated. This is reflected in the bottom right in green color.

\subsection{Variants of the approach}

The heuristic approach is split into two variants: the "restrictive fix-andrun" form and the "conservative fix-and-run" form. The former utilizes the information obtained from a single combination, while the latter utilizes the combined information from all examined combinations.

\subsubsection{Restrictive "fix-and-run"}

For each of the examined combinations, the entire profile of each SL is estimated. The estimated profile is fixed (in the MP-OPF formulation) and the full NLP approximation solved. At this stage, the behavior of all SLs is parameter rather than a variable. This setup offers no flexibility to the solver to re-position the SL in a different way. This form is referred to as the "restrictive fix-and-run", due to the fact that while optimization is still being performed (controllable PV curtailment and import/export schedule), the SL no longer participates in determining the optimal solution.

\subsubsection{Conservative "fix-and-run}

To deal with potential sub-optimality that is inherent in restrictive heuristic approaches based on a single combination, the "conservative fix-and-run" form is also proposed. It is more flexible because it targets only common elements of all examined combinations. Rather than fixing the entire profile for SLs, the focus is solely on the common characteristics of each combination. As such, the pattern of each SL is only partially fixed before solving the NLP approximation (where all remaining formerly binary variables will be forced to be virtually equal to 1 or 0 . The solver is offered some flexibility, increasing its chances of obtaining a better solution as compared to restrictive heuristic approaches. 


\subsection{Quality evaluation}

The quality of an approach depends on the optimality, solution speed and modelling accuracy. To balance all three aspects, we propose a flexible scoring function which provides insight on the quality of the final solution. Every final version $F V$ is benchmarked against the MINLP formulation (denoted by $M I$ ) with respect to the objective function $(f)$, the solution speed $(\Delta t)$ and the accurate representation of SL behavior $(L S)$. Flexible weights $(w)$ are assigned to the three aspects, depending on the needs of the problem. The scoring function is described by $(21)$, where $Q^{\text {sol }}$ is the approach's score:

$$
Q^{s o l}=w_{f} \cdot \frac{f^{M I}}{f^{F V}}+w_{\Delta t} \cdot \frac{\Delta t^{M I}}{\Delta t^{F V}}+w_{L S} \cdot \frac{L S^{M I}}{L S^{F V}}
$$

If there is no benchmark, i.e., no MINLP convergence, appropriately large values are used for $f^{o r}, \Delta t^{o r}$, while $L S^{o r}$ is ignored. Do note that $Q^{\text {sol }}$ is case-dependent, and thus comparisons between different problem setups is not meaningful.

\section{Case studies}

\subsection{Testbed and modelling choices}

The proposed approach is evaluated on two feeders and three optimization horizons $(T=6,12,24)$. An 18-node feeder (Fig. 2, original network available in [23]) is used for proof-of-concept validation. A 308-node feeder (see [24]) is used for tractability evaluation. Nodes with controllable elements are assumed to host SBs. As explained, for SBs with deferable loads (e.g., washers, dryers, heating/cooling loads), they are grouped together into a single SL group, in order to handle them more efficiently in the MP-OPF setting. The problem's specifications are presented in Table 2. PV, SL, load profiles, and feeder technical characteristics are available in [18], [24].

The selected scoring weights are $w_{f}=0.55, w_{\Delta t}=0.4, w_{L S}=0.05$. The NLP, MINLP problems are solved through GAMS [25], using IPOPT [26] and BONMIN (with a defined optimality gap of zero). [27].

Finally, it must be mentioned that the authors' first pathway was solving the MINLP formulation with relaxed binary variables and round their results; this is a common approach for MINLP problems. However, the discrete formulation pre-assumes that $\delta_{s, t}$ are binary. When relaxed, the formulation proved unreliable, returning no discernible SL patterns. 


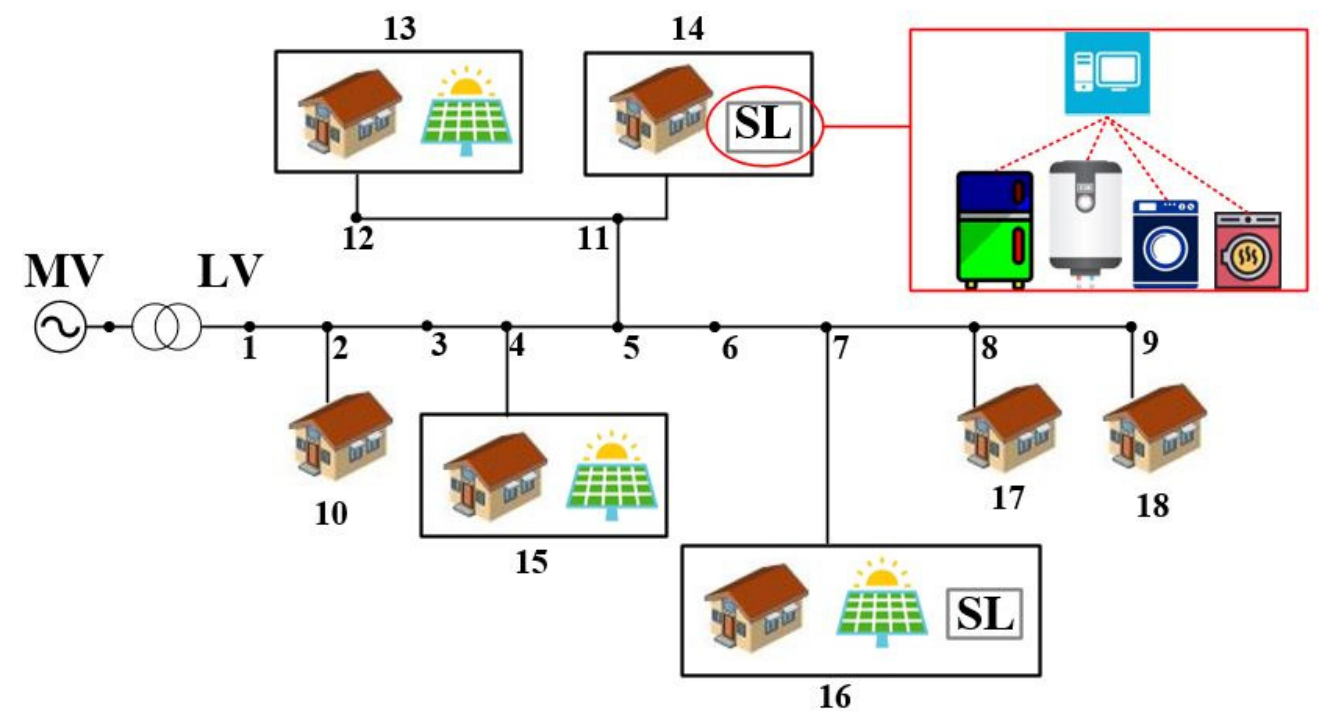

Figure 2: One-line diagram of the 18 node feeder

Table 2: Characteristics of examined feeders

\begin{tabular}{|c|c|c|c|c|c|c|}
\hline \multicolumn{3}{|c|}{ Feeder data } & \multicolumn{3}{c|}{ MP-OPF data $(T=24)$} \\
\hline Size (nodes) & Loads & SLs & PVs & CV & BV & Constraints \\
\hline 18 nodes & 6 & 2 & 3 & 2,520 & 48 & 3,364 \\
308 nodes & 45 & 9 & 16 & 52,056 & 216 & 74,610 \\
\hline
\end{tabular}

${ }^{*} \mathrm{CV}$ : Continuous variables, BV: Binary variables

\subsection{Results: 18-node System}

To understand the main objective and control actions, we first illustrate the physical representation of the optimization process. The unoptimized voltage profiles are presented in Fig. 3. High consumption causes serious undervoltages (as low as $0.8 \mathrm{pu}$ ), leading to stiff violation penalties. While not shown, it is mentioned that the original SL profiles do not overlap significantly with PV production, forcing the curtailment of PV production (curtailment costs $428 €$ ). These observations indicate that SLs should be moved to a different operating horizon.

The optimal operation (MINLP solution) of SLs is presented in Fig. 4. Both SLs are activated earlier, alleviating periods of previously high consumption and no longer contributing to undervoltages. In addition, SL op- 


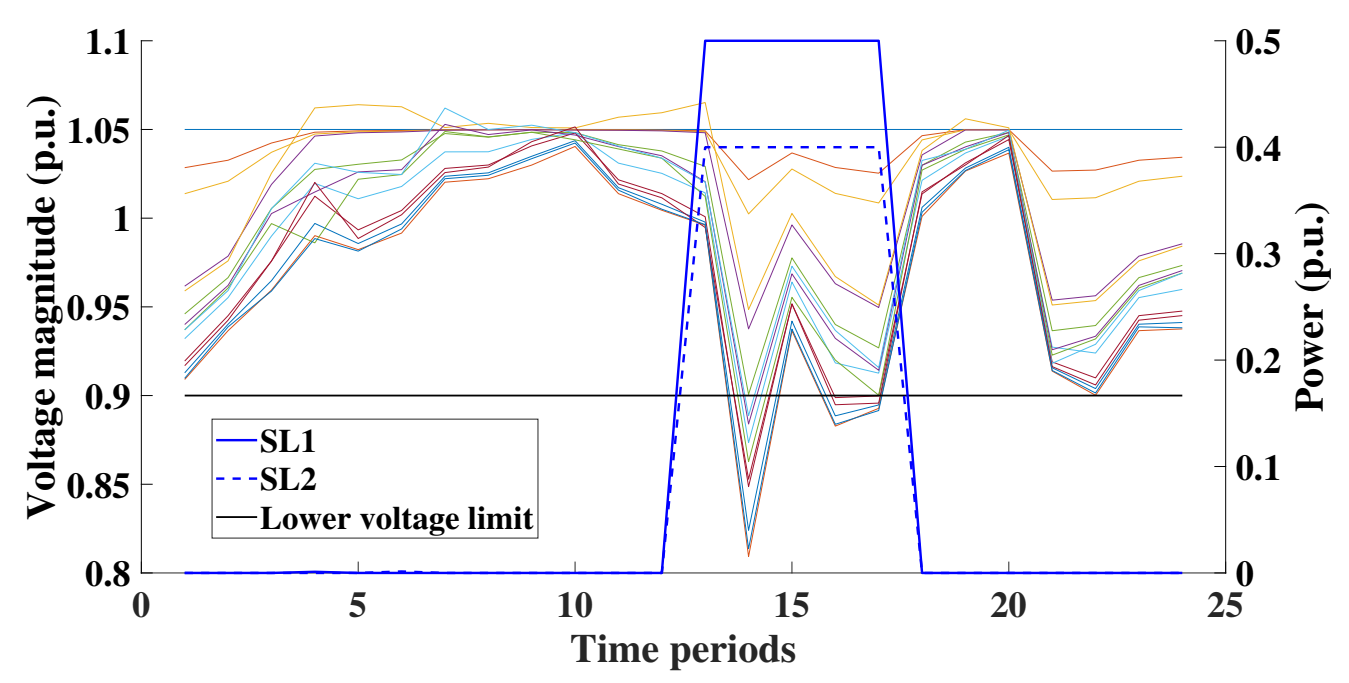

Figure 3: Voltage profiles without SL optimization, 18-node feeder, $T=24, C T=5$ (PV curtailment cost: $428 €$ )

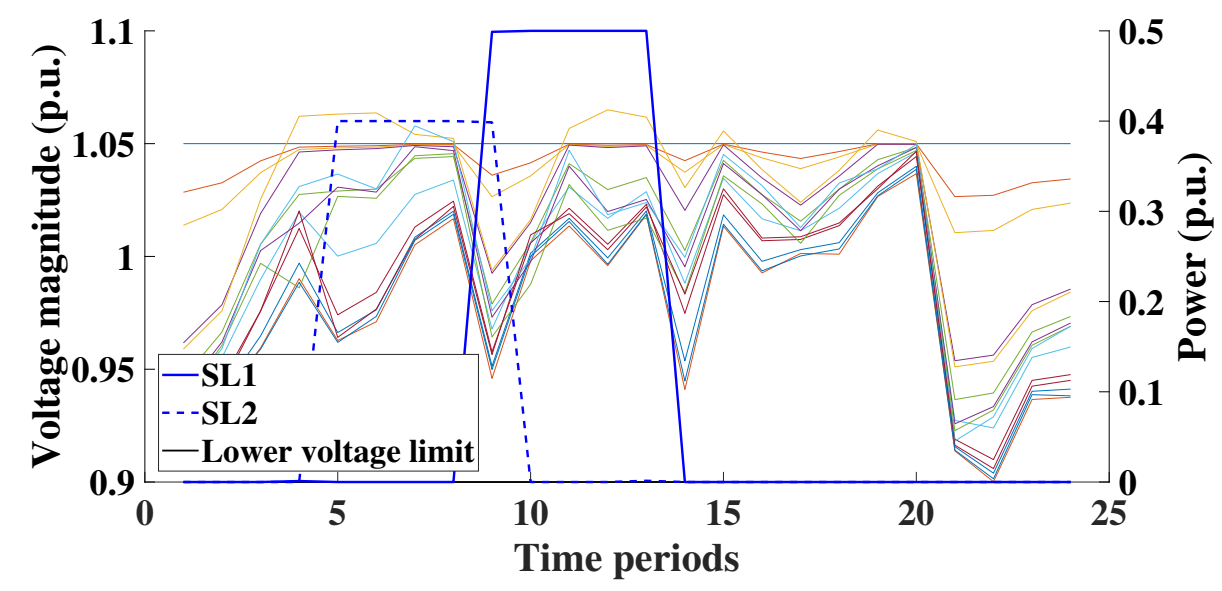

Figure 4: Voltage profiles with SL optimization, 18-node feeder, $T=24, C T=5$ (PV curtailment cost: $382 €$ )

eration now overlaps more with PV production, with the some curtailment actions being avoided (curtailment costs $382 €$ ). What remains is determining the best strategy towards reaching an equally acceptable solution, other than solving the MINLP problem.

The remaining results for the 18-node system are available in Table 3, 
Table 3: Results, 18-node feeder

\begin{tabular}{|c|c|c|c|c|c|c|c|c|c|c|c|c|c|c|c|}
\hline & \multicolumn{3}{|c|}{$T=6$} & \multicolumn{5}{|c|}{$T=12$} & \multicolumn{4}{c|}{$T=24$} & $T \in\{6,12,24\}$ \\
\hline Approach & Obj (€) & Time (s) & $Q^{\text {sol }}$ & Rank & Obj (€) & Time (s) & $Q^{\text {sol }}$ & Rank & Obj (€) & Time (s) & $Q^{\text {sol }}$ & Rank & Average rank \\
\hline MINLP & 105.5 & 11.6 & 100 & 10 & 228.8 & 89.53 & 100 & 10 & 463.3 & 146.9 & 100 & 5 & $8.3 \rightarrow 9$ \\
\hline NLP (1) & 106.2 & 1.9 & 246.6 & 3 & 226.4 & 2.41 & $1,185.2$ & 1 & - & - & 0 & 10 & $4.7 \rightarrow 3$ \\
\hline ES (4) & 113.0 & 7.2 & 113.6 & 9 & 228.8 & 23.62 & 183.7 & 7 & 463.3 & 230.2 & 89.1 & 9 & $8.3 \rightarrow 9$ \\
\hline C (2) & 104.7 & 1.7 & 275.0 & 2 & 253.8 & 6.76 & 460.6 & 4 & 463.3 & 59.1 & 144.5 & 3 & $3 \rightarrow 3$ \\
\hline A (2) & 105.5 & 1.3 & 343.4 & 1 & 228.8 & 3.44 & 850.1 & 3 & 463.3 & 53.7 & 152.1 & 2 & $2 \rightarrow 1$ \\
\hline D (2) & 113.0 & 2.3 & 217.9 & 5 & 253.8 & 11.23 & 302.2 & 5 & 463.3 & 66.5 & 136.3 & 4 & $4.7 \rightarrow 4$ \\
\hline CAD (4) & 113.0 & 6.3 & 121.2 & 7 & 253.8 & 22.42 & 182.9 & 8 & 463.3 & 180.2 & 94.5 & 6 & $7 \rightarrow 8$ \\
\hline \hline ACD (4) & 105.5 & 6.3 & 125.8 & 6 & 253.8 & 22.42 & 182.9 & 8 & 463.3 & 180.2 & 94.5 & 6 & $6.7 \rightarrow 7$ \\
\hline \hline DAC (4) & 113.0 & 6.3 & 121.2 & 7 & 228.8 & 22.42 & 189.8 & 6 & 463.3 & 180.2 & 94.5 & 6 & $6.3 \rightarrow 6$ \\
\hline \hline CONS (4) & 102.0 & 2.1 & 242.7 & 4 & 226.4 & 2.9 & 980.8 & 2 & 463.3 & 4.6 & 1.022 .3 & 1 & $2.3 \rightarrow 2$ \\
\hline
\end{tabular}

*Obj: Objective function value, CONS: Conservative "fix-and-run", - means no convergence or too high solution time

where the performance, the score and rank of each solution approach is presented. In the first column, the number of solved NLP problems per approach is included (SL profile estimation and optimization). For the solution time, the values include SL pattern estimation and subsequent optimization.

While the problem is relatively small, the MINLP formulation returns a solution in reasonable time. However, its performance has a deteriorating trend, being approximately $37.9 \%, 73.6 \%$ and $59.8 \%$ slower than the next faster approach for $T=6,12,24$ respectively. For smaller optimization horizons, the NLP and the "conservative fix-and-run" approaches return accurate solutions with speed improvements of about $96 \%$.

Some approaches calculate better objectives than the MINLP, despite the optimality gap being 0 . This can be attributed to the non-convexity of the MINLP formulation and the BONMIN solver implementation which guarantees at least local optimality. Therefore, it is not out of the ordinary for the developed heuristics to achieve improvements.

The MINLP generally ranks low amongst its "competitors", owing to its intractable nature as the optimization horizon grows larger. One also observes the drop in quality of the NLP approach. This is due to its bad performance for $T=24$, when the barrier function-based constraint (16) begins exhibiting numerical issues. The remainder of the heuristic approaches demonstrate fluctuations in their performance, with the exception of the three original versions $(\mathrm{C}, \mathrm{A}, \mathrm{D})$. The simplest approaches consistently achieve high rankings, with approach $A$ (no limited activation mandate) seemingly performing best. The "conservative fix-and-run" approach achieves favorable scores, indicating that it could also be an effective alternative. 


\subsection{Results: 308-node System}

The same results are presented in Table 4 for the 308-node system. For a more realistic problem, major differences are observed. Apart from the MINLP approach not returning a solution within reasonable time, two approaches initially thought of as effective (NLP and "conservative fix-andrun") have not delivered for $T=12,24$. The size of the problem has caused them to present numerical issues, being ultimately inadequate. On the other hand, the heuristic approaches provide a feasible solution within reasonable time ( $\sim 11$ minutes on average).

Direct benchmarking against the MINLP is difficult for $T=24$; the latter did not produce a solution even after 5 hours. The simplest of the heuristic approaches provide better (and feasible) solutions at a much faster rate (on average, approximately $56 \%, 81 \%$ and $45 \%$ faster respectively) for $T=6,12$. They are ranked second, first and third respectively.

On average, the MINLP ranks dead-last amongst all approaches, either producing no solution or returning a worse solution much slower. The NLP approximation appears to be unsuitable for large-scale problems. The "conservative fix-and-run" approach ranks average, performing well for smaller optimization horizons, but encountering numerical issues for extended ones. The performance of most heuristics exhibits small fluctuations; however, the three simplest techniques maintain their previous status as good alternatives. This consistency is indicative that heuristic solutions relying on relaxing individual SL characteristics are efficient in terms of returning quick, feasible solutions to otherwise difficult problems.

\subsection{Remarks}

Modifying the power system, the optimization horizon or number of SLs affects the performance of each approach on a per-case basis. However, if

Table 4: Results, 308-node feeder

\begin{tabular}{|c|c|c|c|c|c|c|c|c|c|c|c|c|c|c|c|}
\hline & \multicolumn{4}{|c|}{$T=6$} & \multicolumn{4}{c|}{$T=12$} & \multicolumn{4}{c|}{$T=24$} & $T \in\{6,12,24\}$ \\
\hline Approach & Obj (€) & Time (s) & $Q^{\text {sol }}$ & Rank & Obj (€) & Time (s) & $Q^{\text {sol }}$ & Rank & Obj (€) & Time (s) & $Q^{\text {sol }}$ & Rank & Average rank \\
\hline MINLP & 520.7 & 237.5 & 100 & 10 & 864.1 & $2,012.7$ & 100 & 9 & - & - & 100 & 8 & $9 \rightarrow 10$ \\
\hline NLP (1) & 598.2 & 37.8 & 249.3 & 3 & - & - & 0 & 10 & - & - & 100 & 8 & $7 \rightarrow 7$ \\
\hline ES (4) & 531.7 & 145.8 & 117.4 & 7 & 593 & 523.0 & 217.5 & 5 & $2,071.5$ & 633.2 & 811.7 & 4 & $5.3 \rightarrow 5$ \\
\hline C (2) & 585.5 & 40.9 & 236.4 & 4 & 558 & 318.8 & 297.8 & 3 & $1,850.3$ & 285.6 & $1,482.9$ & 2 & $3 \rightarrow 2$ \\
\hline A (2) & 527.0 & 23.3 & 375.3 & 1 & 745.2 & 145.0 & 497.5 & 1 & $1,955.6$ & 119.9 & $2,860.9$ & 1 & $1 \rightarrow 1$ \\
\hline D (2) & 561.1 & 70.3 & 166.3 & 5 & 802.2 & 228.3 & 339.9 & 2 & $2,023.1$ & 358.1 & $1,183.9$ & 3 & $3.3 \rightarrow 3$ \\
\hline CAD (4) & 537.6 & 145.8 & 116.7 & 8 & 742.0 & 472.0 & 209.4 & 7 & $2,122.0$ & 642.9 & 796.5 & 6 & $7 \rightarrow 7$ \\
\hline \hline ACD (4) & 527.0 & 145.8 & 118.0 & 6 & 742.8 & 455.4 & 214.0 & 6 & $2,079.4$ & 641.0 & 804.6 & 5 & $5.7 \rightarrow 6$ \\
\hline \hline DAC (4) & 537.6 & 145.8 & 116.7 & 8 & 638.5 & 561.9 & 202.2 & 8 & $2,010.2$ & 670.8 & 795.4 & 7 & $7.7 \rightarrow 9$ \\
\hline \hline CONS (4) & 541.0 & 29 & 313.0 & 2 & 687.8 & 463.5 & 218.2 & 4 & - & - & 100 & 8 & $4.7 \rightarrow 4$ \\
\hline
\end{tabular}


one examines the average performance of each approach across all test cases, solid conclusions can be drawn based on some consistent observations:

- The MINLP approach is impractical. It consistently ranks very low amongst all other approaches.

- The NLP approximation approach ranks relatively low. While it performs adequately for smaller problems, it is unsuitable when the number of variables grows too large.

- The "conservative fix-and-run" approach demonstrates a relatively good performance, owing to its ability of providing some flexibility to the solver. It tends to under-perform when the problem size is too large or when only a small percentage of the SLs' behavior can be "fixed".

- The three simplest versions of the heuristic approach, i.e., those based on relaxing individual SL characteristics, are excellent alternatives to solving the original problem, returning fast, and most importantly, feasible solutions.

\section{Conclusions and outlook}

This work tackles a key issue that is under-addressed in the scientific literature, namely the management of high shares of SLs (20\%-30\% of customer nodes in this work) in MP-OPF formulations.

The paper proposes a novel NLP approximation for MP-OPF problems that include shiftable loads and subsequently complements it with a multiversion heuristic technique to efficiently handle large-scale MINLP MP-OPF problems with high presence of shiftable loads. The MINLP approach was shown to essentially be a non-option when the problem size grows substantially. The proposed alternative approaches were shown to be scalable, providing fast and accurate solutions in the vast majority of examined cases. The proposed ranking scheme, while simple, presents consistent results regarding the performance of each approach for different problem sizes.

It is worth stating that the paper makes no claim on the universal superiority of any single approach, but rather presents the strengths and weaknesses of several heuristics and approximations, as well as provides a simple quality evaluation framework. Depending on the needs of each researcher, 
the suitability of each approach can be separately evaluated and appropriate modifications can be made. Said modification are easy, given that the proposed approaches are generic enough in their construction.

In the future, a validation to unbalanced distribution systems will be carried out. In addition, new SL models will be proposed for MP-OPF studies that represent different realistic behaviors, such as the representation of a fixed time series.

\section{Acknowledgments}

The authors acknowledge the funding from the Luxembourg National Research Fund (FNR) in the framework of the gENESiS project (C18/SR/12676686).

\section{References}

[1] S.M. Amin and B.F. Wollenberg. "Toward a smart grid: power delivery for the 21st century". IEEE Power Energy Mag., 3(5):34-41, 2012.

[2] I.I. Avramidis, V.A. Evangelopoulos, P.S. Georgilakis, and N.D. Hatziargyriou. "Demand side flexibility schemes for facilitating the high penetration of residential distributed energy resources". IET Gener. Transm. Distr., 12(18):4079-4088, 2018.

[3] S. Hanif, T. Massier, H.B. Gooi, et al. "Cost optimal integration of flexible buildings in congested distribution grids". IEEE Trans. Power Syst., 32(3):2254-2266, 2016.

[4] G. O'Brien and R. Rajagopal. "Scheduling non-preemptive deferrable loads". IEEE Trans. Power Systems, 31(2):835-845, 2015.

[5] A.-H. Mohsenian-Rad and A. Leon-Garcia. "Optimal residential load control with price prediction in real-time electricity pricing environments". IEEE Trans. Smart Grid, 1(2):120-133, 2010.

[6] M.R. Sarker, M.A. Ortega-Vazquez, and D.S. Kirschen. "Optimal coordination and scheduling of demand response via monetary incentives". IEEE Trans. Smart Grid, 6(3):1341-1352, 2015.

[7] J.E. Contreras-Ocana, M.R. Sarker, and M.A. Ortega-Vazquez. "Decentralized coordination of a building manager and an electric vehicle aggregator". IEEE Trans. Smart Grid, 9(4):2625-2637, 2018. 
[8] A. Papavasiliou and S.S. Oren. "Supplying renewable energy to deferrable loads: algorithms and economic analysis". In IEEE PES General Meeting, July, 2010.

[9] C. Li, D. Srinivasan, and T. Reindl. "Real-time scheduling of timeshiftable loads in smart grid with dynamic pricing and photovoltaic power generation". In ISGT Asia, November, 2015.

[10] M.C. Vlot, J.D. Knigge, and J.G. Slootweg. "Economical regulation power through load shifting with smart energy appliances". IEEE Trans. Smart Grid, 4(3):1705-1712, 2013.

[11] R. Thapa, L. Jiao, B.J. Oommen, and A. Yazidi. "A Learning automaton-based scheme for scheduling domestic shiftable loads in smart grids". IEEE Access, 6:5348-5361, 2017.

[12] I. Tinnirello, G. Neglia, L. Giarre, et al. "Large scale control of deferrable domestic loads in smart grids". IEEE Trans. Smart Grid, 9(2):733-742, 2018 .

[13] K.S. Stille, J. Bocker, N. Frohleke, et al. "Local grid load coordination for load-shiftable domestic appliances in a variable-tariff environment". In European Conf. Power Electronics and Applications, September, 2016.

[14] F. Bliman, A. Ferragut, and F. Paganini. "Controlling aggregates of deferrable loads for power system regulation". In American Control Conf., July, 2015.

[15] R. Cakmak and I.H. Altas. "Scheduling of domestic shiftable loads via cuckoo search optimization algorithm". In ICSG, April, 2016.

[16] Q. Dong, D. Niyato, P. Wang, and S. Gong. 'Deferrable load scheduling of smart meter with intermittent communication connection". In IEEE SmartGridComm, November, 2012.

[17] S. Gill, I. Kockar, and G.W. Ault. "Dynamic optimal power flow for active distribution networks". IEEE Trans. Power Syst., 29(1):121-131, 2014. 
[18] I.I. Avramidis, F. Capitanescu, and G. Deconinck. "A tractable approximation approach for dealing with the binary nature of shiftable loads in multi-period optimal power flow". In TPEC, February, 2020.

[19] T. Soares, R.J. Bessa, P. Pinson, H. Morais. "Active distribution grid management based on robust AC optimal power flow". IEEE Trans. Smart Grid., 9(6):6229-6241, 2018.

[20] O. Erdinc, A. Tascikaraoglou, N. Paterakis, et al. "End-user comfort oriented Day-ahead planning for responsive residential HVAC demand aggregation considering weather forecasts". In IEEE Power \& Energy Society General Meeting, July 2017.

[21] S. Lilla, C. Orozco, A. Borghetti, et al. "Day-ahead scheduling of a local energy community: An alternating direction method of multipliers approach". IEEE Trans. Power Systems, 35(2):1132-1142, 2020.

[22] V.A. Evangelopoulos, I.I Avramidis, and P.S. Georgilakis. "Flexibility services management under uncertainties for power distribution systems: Stochastic scheduling and predictive real-time dispatch". IEEE Access, 8, 2020 .

[23] K. Strunz, E. Abbasi, R. Fletcher, et al. "Benchmark Systems for Network Integration of Renewable and Distributed Energy Resources". CIGRE Task Force C6.04, 4:4-6, 2014.

[24] A. Navarro-Espinosa and L.F. Ochoa. "Probabilistic impact assessment of low carbon technologies in LV distribution systems". IEEE Trans. Power Syst., 31(3):2192-2203, 2015.

[25] B.A. McCarl, GAMS user guide, version 23.8, 2012, www.gams.com.

[26] A. Wächter and L. T. Biegler. "On the implementation of a primaldual interior point filter line search algorithm for large-scale nonlinear programming". Mathematical Programming, 106(1):25-57, 2006.

[27] P. Bonami et al. "An algorithmic framework for convex mixed integer nonlinear programs". Discrete Optimization, 5(2):186-204, 2008. 